\title{
N-Benzoimidazole/Oxadiazole Hybrid Universal Electron Acceptors for Highly Efficient Exciplex-Type Thermally Activated Delayed Fluorescence OLEDs
}

\author{
Wenbo Yuan ${ }^{1 \dagger}$, Hannan Yang ${ }^{2 \dagger}$, Mucan Zhang ${ }^{1}$, Die Hu ${ }^{1}$, Ning Sun ${ }^{2 *}$ and Youtian Tao ${ }^{1 *}$ \\ 1 Key Lab for Flexible Electronics and Institute of Advanced Materials (IAM), Nanjing Tech University, Nanjing, China, \\ ${ }^{2}$ Department of Physics, Yunnan University, Kunming, China
}

OPEN ACCESS

Edited by:

Lian Duan

Tsinghua University, China

Reviewed by:

Guohua Xie,

Wuhan University, China

CaiJun Zheng,

University of Electronic Science and

Technology of China, China

${ }^{*}$ Correspondence:

Ning Sun

ning.sun@ynu.edu.cn

Youtian Tao

iamyttao@njtech.edu.cn

tThese authors have contributed equally to this work

Specialty section

This article was submitted to

Organic Chemistry,

a section of the journal

Frontiers in Chemistry

Received: 17 January 2019

Accepted: 11 March 2019

Published: 03 April 2019

Citation:

Yuan $W$, Yang $H$, Zhang $M$, Hu D,

Sun $N$ and Tao Y (2019)

N-Benzoimidazole/Oxadiazole Hybrid

Universal Electron Acceptors for

Highly Efficient Exciplex-Type

Thermally Activated Delayed

Fluorescence OLEDs.

Front. Chem. 7:187.

doi: 10.3389/fchem.2019.00187
Recently, donor/acceptor type exciplex have attracted considerable interests due to the low driving voltages and small singlet-triplet bandgaps for efficient reverse intersystem crossing to achieve 100\% excitons for high efficiency thermally activated delayed fluorescence (TADF) OLEDs. Herein, two $N$-linked benzoimidazole/oxadiazole hybrid electron acceptors were designed and synthesized through simple catalyst-free C-N coupling reaction. 24iPBIOXD and ITPBIOXD exhibited deep-blue emission with peak at 421 and $459 \mathrm{~nm}$ in solution, 397 and $419 \mathrm{~nm}$ at film state, respectively. The HOMO/LUMO energy levels were $-6.14 /-2.80$ for 24 PPBIOXD and $-6.17 /-2.95 \mathrm{eV}$ for $\mathrm{TPBIOXD}$. Both compounds could form exciplex with conventional electron donors such as TAPC, TCTA, and $\mathrm{mCP}$. It is found that the electroluminescent performance for exciplex-type OLEDs as well as the delayed lifetime was dependent with the driving force of both $\mathrm{HOMO}$ and LUMO energy offsets on exciplex formation. The delayed lifetime from 579 to 2,045 ns was achieved at driving forces close to or larger than $1 \mathrm{eV}$. Two TAPC based devices possessing large HOMO/LUMO offsets of 1.09-1.34 eV exhibited the best EL performance, with maximum external quantum efficiency (EQE) of $9.3 \%$ for 24 iPBIOXD and $7.0 \%$ for $I \mathrm{TPBIOXD}$ acceptor. The TCTA containing exciplex demonstrated moderate energy offsets (0.88-1.03 eV) and EL efficiency ( 4\%), while mCP systems showed the poorest EL performance (EQE $<1 \%$ ) and shortest delayed lifetime of $<100$ ns due to inadequate driving force of $0.47-0.75 \mathrm{eV}$ for efficient exciplex formation.

Keywords: electron acceptor, electron donor, exciplex, oxadiazole, thermally activated delayed fluorescence, OLEDs

\section{INTRODUCTION}

Organic light-emitting diodes (OLEDs) have been developed rapidly in recent years since the pioneer work on low-voltage fluorescence electroluminescence by Tang in 1987 (Tang and VanSlyke, 1987; Ma et al., 1998; Gong et al., 2010; Park et al., 2013; Zhang et al., 2016). According to spin statistics, the ratio for singlet and triplet excitons recombined from electrogenerated holes and electrons is 1:3 (Baldo et al., 1999; Segal et al., 2003). Thus, the first generation of traditional fluorescent OLEDs which solely harvest singlet excitons only shows $25 \%$ of maximum internal 
quantum efficiency (IQE) (Wen et al., 2005). On the other hand, the second generation of phosphorescent OLEDs (PHOLEDs) based on heavy metal complexes and third generation of thermally activated delayed fluorescence (TADF) OLEDs could both reach $100 \%$ IQE in theory by utilizing all singlet and triplet excitons through intersystem crossing (ISC) and reverse inter-system crossing (RISC), respectively (Baldo et al., 1998; Adachi et al., 2001; Su et al., 2008; Lo et al., 2009; Goushi et al., 2012; Uoyama et al., 2012; Zhang and Forrest, 2012; Li et al., 2016; Cao et al., 2017; Huang et al., 2018; Wu Q. et al., 2018). However, to avoid consuming noble metals and achieving reliable true-blue light, TADF OLEDs based on low-cost pure organic emitters have attracted increasing interests as an alternative mechanism to PHOLEDs. TADF emission is realized by an upconversion process from lower energy triplet states to slightly higher energy singlet states by endothermic reverse inter-system crossing process ( $\mathrm{Li}$ et al., 2016; Cao et al., 2017; Huang et al., 2018; Wu Q. et al., 2018). Therefore, a small singlet-triplet energy bandgap $\left(\Delta E_{\mathrm{ST}}\right)$ is required for TADF emitters.

It is reported that the small $\Delta E_{\mathrm{ST}}$ could be attained in (i) intramolecular charge transfer featured single molecule with twisted donor-acceptor structured for effective spatial isolation between the highest occupied molecular orbital (HOMO) and lowest unoccupied molecular orbital (LUMO) on the relevant hole and electron transporting moieties, and (ii) bimolecularexciplex which contains an electron-donor material mixed with an electron-acceptor material through intermolecular charge transfer characteristics (Cai and Su, 2018; Liu et al., 2018; Sarma and Wong, 2018). High external quantum efficiency (EQE) of $20 \%$ for red, $29 \%$ for orange, $38 \%$ for green, and $37 \%$ for lightblue TADF OLEDs have been achieved in single-molecule TADF emitters (Lin et al., 2016; Chen et al., 2018; Wu T.-L. et al., 2018; Zeng et al., 2018). However, the development of bimolecular TADF lags far behind. Most exciplex-type TADF OLEDs showed maximum EQE close to 10\% (Jankus et al., 2014; Liu et al., 2015a,b; Oh et al., 2015; Zhang L. et al., 2015; Hung et al., 2016, 2017; Jeon et al., 2016), with only one example approaching to 18\% (Liu et al., 2016).

In electron donor/acceptor formed exciplex systems, compared with commercially available various electron-donor materials, such as $4,4^{\prime}, 4^{\prime \prime}$-tris[3-methylphenyl(phenyl)amino]triphenylamine (m-MTDATA), $\quad N, N^{\prime}$-bis(1-naphthyl)$N, N^{\prime}$-diphenyl-[1,1'-biphenyl]-4, $4^{\prime}$-diamine $4,4^{\prime}$-(cyclohexane-1,1-diyl)bis ( $N$-phenyl-N-p-tolylaniline) (TAPC), 4, $4^{\prime}, 4^{\prime \prime}$-tris(N-carbazolyl) triphenylamine (TCTA), $4,4^{\prime}$-bis( $N$-carbazolyl)-1, $1^{\prime}$-biphenyl (CBP), and $N, N^{\prime}$ dicarbazolyl-3,5-benzene (mCP) etc., the types of efficient and low-cost electron-acceptor materials are scarce (Goushi and Adachi, 2012; Goushi et al., 2012; Sun et al., 2014; Lee et al., 2015; Liu et al., 2015b). Thus, the exploration of electron accepting materials is essential for constructing exciplex systems with outstanding optoelectronic performance. Therefore, in this work, we designed and synthesized two new electron-acceptors of 2-(2,4-bis(2-phenyl-1H-benzo[d]imidazol1-yl)phenyl)-5-phenyl-1,3,4-oxadiazole (24iPBIOXD), and 2-phenyl-5-(2,4,6-tris(2-phenyl-1H-benzo[d]imidazol-1-

yl)phenyl)-1,3,4-oxadiazole (iTPBIOXD) through a simple one-step catalyst-free aromatic nucleophilic substitution reaction. The electron-withdrawing oxadiazole (OXD) unit has been extensively applied in donor-acceptor type bipolar transport host materials, single molecule intramolecular charge transfer type TADF emitters as well as electron transport materials (Tao et al., 2011; Mondal et al., 2013; Olivier et al., 2017; Cooper et al., 2018; Yao et al., 2018; Zhang et al., 2018). By combining OXD building block with our previously reported isomeric $N$ linkaged benzoimidazole (Hu et al., 2017), both 24iPBIOXD, and $i$ TPBIOXD exhibited deep HOMO level of $\sim-6.15 \mathrm{eV}$, facilitating the exciplex formation with general electron donor materials of TAPC, TCTA, and mCP due to the compatible HOMO and LUMO energy levels between donor and acceptor materials. The gradient energy offsets ranging from 0.47 to $1.34 \mathrm{eV}$ correlated well with the delayed lifetime and EL efficiencies in exciplex type TADF OLEDs. The TAPC:24iPBIOXD exciplex with the largest HOMO/LUMO offsets exhibited the best EL performance, with maximum EQE of $9.3 \%$ for green TADF OLEDs.

\section{RESULTS AND DISCUSSION}

\section{Synthesis and Characterization}

Scheme 1 shows the synthetic routes and molecular structures of 24iPBIOXD and $i$ TPBIOXD. The two compounds could be facilely synthesized by a simple one-step catalyst free C-N coupling reaction. This nucleophilic substitution reaction was carried out in DMSO solvent with $\mathrm{K}_{2} \mathrm{CO}_{3}$ base at high yields over $80 \%$ by using di/tri-fluorine substituted oxadiazole derivatives as electrophiles and 2-phenyl-1H-benzo[d]imidazole as nucleophiles. The considerably high yields and environmentally eco-friendly conditions demonstrated the superiority than common metal-catalyzed Ullman reactions (Son et al., 2008; Liu et al., 2011; Volz et al., 2013). In addition, the directly connection of the isomeric $N$-linked benzoimidazole to the central phenyl ring avoided the complicated multistep ring-closing synthetic process for the normal $C$-linked benzoimidazole in traditional electron transport material of 2,2,2-(1,3,5phenylene)-tris(1-phenyl-1H-benzoimidazole) (TPBI) or its derivatives. The chemical structures of the new compounds were fully characterized by ${ }^{1} \mathrm{H} \mathrm{NMR},{ }^{13} \mathrm{C}$ NMR, mass spectrometry (MALDI-TOF) and element analysis (Figure S1). The good thermal stability of the two compounds was confirmed by thermogravimetric analysis (TGA) and differential scanning calorimetry (DSC) (Figure 1). The decomposition temperatures ( $T_{\mathrm{d}}$, corresponding to a $5 \%$ weight loss) from TGA curves for $24 i \mathrm{PBIOXD}$ and $i$ TPBIOXD were determined at 443 and $461^{\circ} \mathrm{C}$, respectively. Additionally, the melting point $\left(T_{\mathrm{m}}\right)$ of $i \mathrm{TPBIOXD}$ was observed at $327^{\circ} \mathrm{C}$, which was much higher than $286^{\circ} \mathrm{C}$ of $24 i \mathrm{PBIOXD}$. The glass transition temperature $\left(T_{\mathrm{g}}\right)$ of both materials can be detected from the second heating cycles from DSC, with values of $126^{\circ} \mathrm{C}$ for $24 i \mathrm{PBIOXD}$ and $165^{\circ} \mathrm{C}$ for $i \mathrm{TPBIOXD}$, indicating their reasonable thermal stability.

\section{Photophysical Properties}

The room temperature UV-Vis absorption and photoluminescence (PL) spectra of 24iPBIOXD and $i$ TPBIOXD 

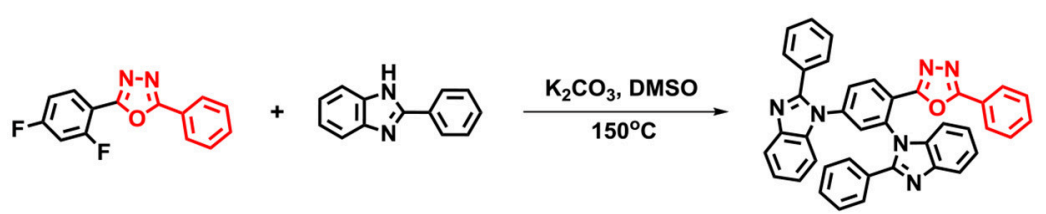

24IPBIOXD
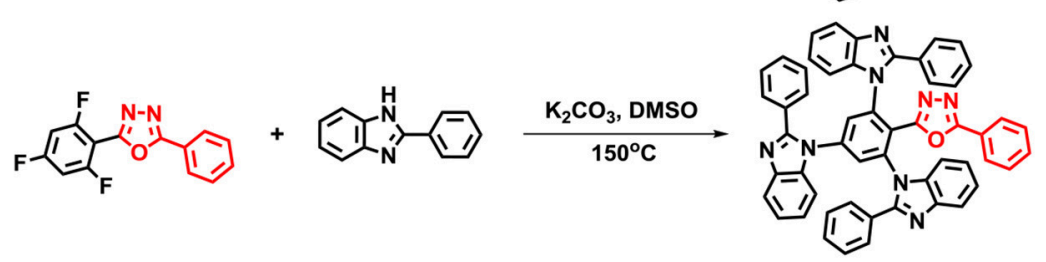

TTPBIOXD

SCHEME 1 | Synthesis of compounds 24ITPBIOXD and ITPBIOXD.
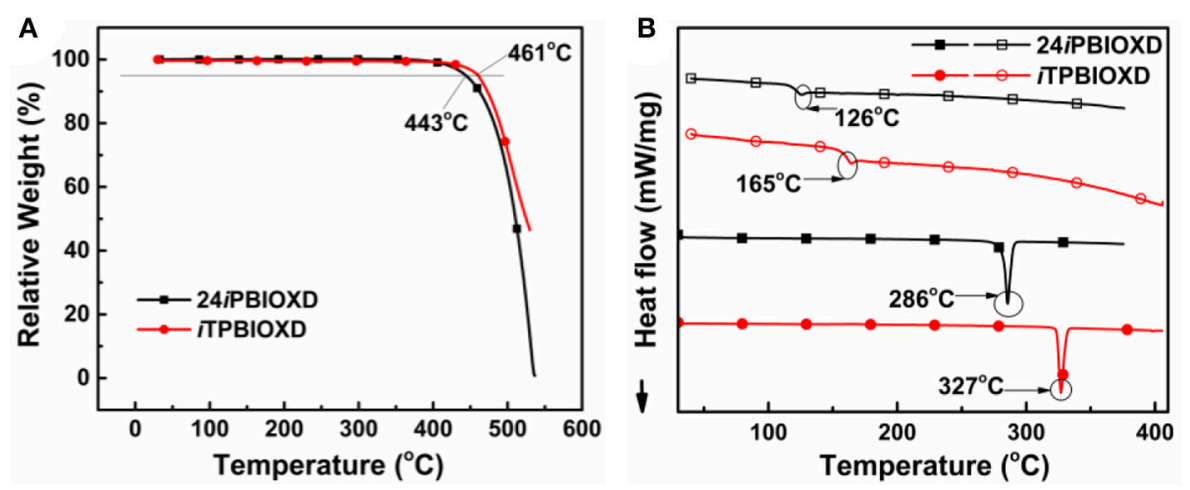

FIGURE 1 | (A) Thermogravimetric analysis (TGA) and (B) differential scanning calorimetry (DSC) (solid symbols represent for first heating scan and open symbols for second heating scan) curves for 24iPBIOXD and ITPBIOXD.

in $\mathrm{CH}_{2} \mathrm{Cl}_{2}$ solution are shown in Figure 2A. Both compounds exhibited an intense absorption with peaks at 289 and $283 \mathrm{~nm}$ in solution, 297 and $288 \mathrm{~nm}$ in film, respectively, which can be ascribed to the $\pi-\pi^{*}$ transition of molecules. The optical bandgap $\left(E_{\mathrm{g}}\right)$ was calculated to be $3.34 \mathrm{eV}$ for $24 i \mathrm{PBIOXD}$ and $3.22 \mathrm{eV}$ for $i$ TPBIOXD, according to the film-state absorption edge. On the other hand, 24iPBIOXD and $i$ TPBIOXD showed unimodal photoluminescence peaking at 421 and $459 \mathrm{~nm}$ in solution, whereas significantly blue-shift to 397 and $419 \mathrm{~nm}$ in film state (Table 1). By analyzing the highest-energy vibronic sub-band of low-temperature fluorescence and phosphorescence spectrum (Figure 2B), the singlet $\left(E_{\mathrm{S}}\right)$ and triplet $\left(E_{\mathrm{T}}\right)$ energy levels could be determined to be $3.31 / 2.55$ and $3.18 / 2.53 \mathrm{eV}$ for $24 i \mathrm{PBIOXD}$ and $i \mathrm{TPBIOXD}$, respectively. In addition, the $E_{\mathrm{S}} / E_{\mathrm{T}}$ energy levels of three hole-transport electron donor materials were also calculated to be $3.54 / 2.95 \mathrm{eV}$ for $\mathrm{mCP}$, $3.79 / 2.82 \mathrm{eV}$ for TAPC, and 3.66/2.84 eV for TCTA (Figure 2C). The PL spectra for the neat film of electron donors such as $\mathrm{mCP}$, TAPC, and TCTA, the two new electron-acceptors of 24iPBIOXD and $i$ TPBIOXD as well as their corresponding mixtures in a 1:1 weight ratio were investigated. As shown in Figure 3 and Figure S2, all blended films showed bathochromic shifted PL spectra compared with the emission of neat $24 i \mathrm{PBIOXD} / i \mathrm{TPBIOXD}$ and the corresponding donor-material, indicating the successful formation of exciplex (Zhang T. et al., 2015). In addition, it is found that exciplex based on 24iPBIOXD acceptors all exhibited about $20-30 \mathrm{~nm}$ blue-shifted emission than $i$ TPBIOXD based exciplex systems. The exciplex emission color could be tuned from deep-blue of mCP:24iPBIOXD with peak at $419 \mathrm{~nm}$ to light-blue of TCTA:24iPBIOXD $(501 \mathrm{~nm})$ and further to green of TAPC:24iPBIOXD $(518 \mathrm{~nm})$. Besides, transient photoluminescence $(\mathrm{PL})$ measurements were carried out for all six exciplexes (Figure 4). The exciplexes comprising TAPC or TCTA donor all possessed significantly longer delayed decay lifetime, with values of 579 ns for TCTA:24iPBIOXD, $1,907 \mathrm{~ns}$ for TCTA:iTPBIOXD, 1,520 and 2,045 ns for TAPC:24iPBIOXD, TAPC:iTPBIOXD exciplex, respectively. However, the mCP:24iPBIOXD and mCP:iTPBIOXD exciplex systems displayed greatly shorter delayed decay lifetime of only 42 and 72 ns (Table 1). Besides, the temperature dependent PL transients for the representative TAPC:24iPBIOXD and TCTA:iTPBIOXD exciplexes (Figure S3) both demonstrated a more significant decay from 100 to $300 \mathrm{~K}$ at the longer lifetime range, suggesting the potential existence of endothermic reverse inter-system crossing. It is expected the obvious variations on delayed decay time for different exciplexes may demonstrate some relationships with the device efficiency in exciplex-TADF OLEDs. 

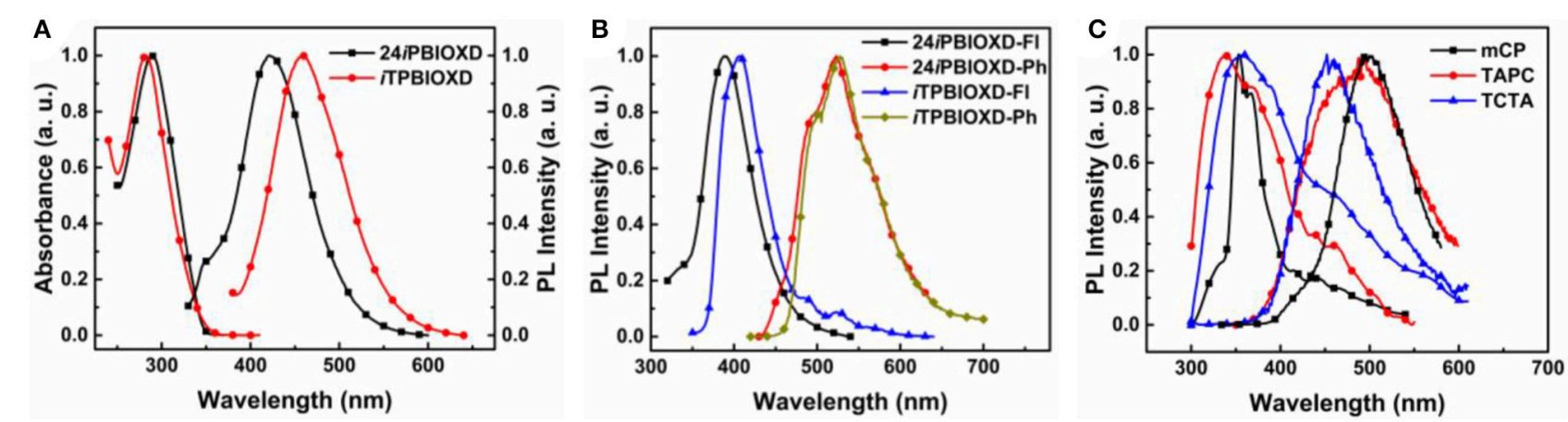

FIGURE 2 | (A) Normalized UV-Vis absorption and PL spectra for 24iTPBIOXD and ITPBIOXD in $\mathrm{CH}_{2} \mathrm{Cl}_{2}$ solution, (B) $77 \mathrm{~K}$ fluorescence (FI) and phosphorescence $(\mathrm{Ph})$ spectra for 24TPBIOXD and ITPBIOXD in neat film, (C) $77 \mathrm{~K}$ fluorescence (Fl) and phosphorescence (Ph) spectra for three donor materials in neat film.

TABLE 1 | Physical properties of compounds 24iPBIOXD and ITPBIOXD.

\begin{tabular}{|c|c|c|c|c|c|c|c|c|c|}
\hline Compounds & $\begin{array}{c}\lambda_{\text {abs }} / \lambda_{\text {em }} \mathrm{a} \\
{[\mathrm{nm}]}\end{array}$ & $\begin{array}{c}\lambda_{\text {abs }} / \lambda_{\text {em }} \mathrm{b} \\
{[\mathrm{nm}]}\end{array}$ & $\begin{array}{c}E_{\mathrm{S}} / E_{\mathrm{T}} \mathrm{c} \\
{[\mathrm{eV}]}\end{array}$ & $\begin{array}{l}E_{\mathrm{g}} \mathrm{d} \\
{[\mathrm{eV}]}\end{array}$ & $\begin{array}{c}\text { HOMO/LUMOe } \\
{[\mathrm{eV}]}\end{array}$ & $\begin{array}{c}T_{\mathrm{g}} / T_{\mathrm{m}} / T_{\mathrm{d}} \mathrm{f} \\
{\left[{ }^{\circ} \mathrm{C}\right]}\end{array}$ & \multicolumn{3}{|c|}{$\lambda_{\mathrm{CT}}(\mathrm{nm}) / E_{\mathrm{CT}}(\mathrm{eV}) / \tau_{\mathrm{d}}(\mathrm{ns}) / \Delta E(\mathrm{eV})^{\mathrm{g}}$} \\
\hline 24iPBIOXD & $289 / 421$ & $297 / 397$ & $3.31 / 2.55$ & 3.34 & $\begin{array}{l}-6.14(-5.75) / \\
-2.80(-2.09)\end{array}$ & $126 / 286 / 443$ & $\begin{array}{c}419 / 2.96 / 42 \\
0.47 / 0.6\end{array}$ & $\begin{array}{c}501 / 2.48 / 579 \\
0.95 / 0.88\end{array}$ & $\begin{array}{c}518 / 2.39 / 1520 \\
1.09 / 1.19\end{array}$ \\
\hline ITPBIOXD & $283 / 459$ & $288 / 419$ & $3.18 / 2.53$ & 3.22 & $\begin{array}{c}-6.17(-5.95) / \\
-2.95(-2.05)\end{array}$ & $165 / 327 / 461$ & $\begin{array}{c}443 / 2.80 / 72 \\
0.5 / 0.75\end{array}$ & $\begin{array}{c}531 / 2.36 / 1907 \\
0.98 / 1.03\end{array}$ & $\begin{array}{c}544 / 2.28 / 2045 \\
1.12 / 1.34\end{array}$ \\
\hline
\end{tabular}

a Measured in $\mathrm{CH}_{2} \mathrm{Cl}_{2}$ solution at room temperature.

${ }^{b}$ Measured in film.

c Singlet energy and triplet energy was calculated from low temperature $(77 \mathrm{~K})$ fluorescence spectra and phosphorescence spectrum.

${ }^{d}$ Optical bandgap $\left(E_{g}\right)$ calculated from the absorption edge of film state UV-Vis spectra.

e LUMO measured from the onset of reduction curves from CV and HOMO calculated from the difference between LUMO and Eg, values in parentheses from DFT calculations.

${ }^{f}$ Glass transition temperature/melting point/decomposition temperature.

${ }^{g}$ Emission maxima, charge transfer state energy, delayed decay lifetime and HOMO/LUMO energy offsets for various exciplexes.
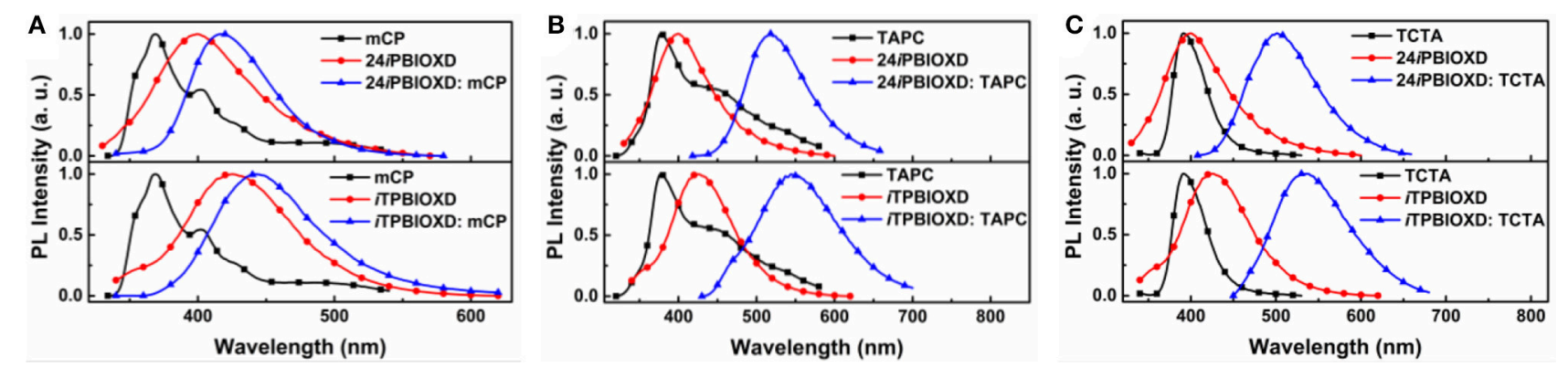

FIGURE 3 | PL spectra of (A) MCP, (B) TAPC, (C) TCTA with different electron acceptors and the relevant exciplexes in film.

\section{Theoretical Calculations and Electrochemical Properties}

In order to gain insights into the frontier molecular orbital and excited states level distribution of 24iPBIOXD and iTPBIOXD, density functional theory (DFT) calculation was conducted at the B3LYP level (Francl et al., 1982; Becke, 1988; Lee et al., 1988). From the optimized geometry shown in Figure 5, the dihedral angles between the central phenyl and oxadiazole ring were 22.0 and $50.3^{\circ}$ for $24 i \mathrm{PBIOXD}$ and $i$ TPBIOXD, respectively, the values between the benzoimidazoles and the central phenyl rings ranged from 50.4 to $77.4^{\circ}$, indicating a twisted structure for both compounds. Furthermore, in the ground state, the highest occupied molecular orbital (HOMO) were almost completely located on one of the ortho-positioned phenylbenzoimidazole units, indicating the electron-donating characteristics of $\mathrm{N}$-linked phenylbenzoimidazole, which was quite different from the $\mathrm{C}$-isomerized phenylbenzoimidazole containing TPBI (Hu et al., 2017). And the lowest unoccupied molecular orbital (LUMO) were mainly localized on 2,5diphenyl-1,3,4-oxadiazole, along with mildly distribution over 

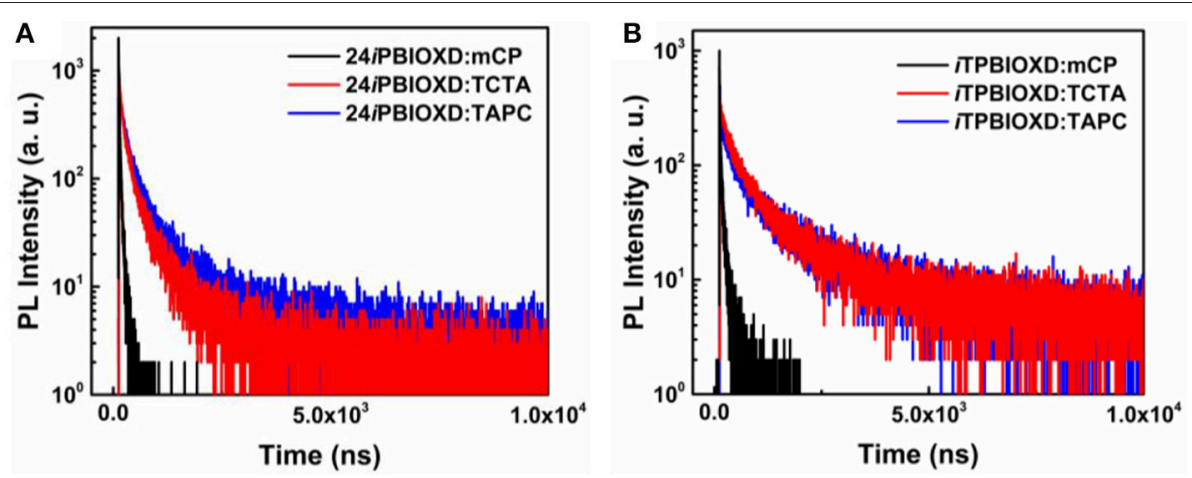

FIGURE 4 | Transient decay curves of (A) 24iPBIOXD and (B) iTPBIOXD-based exciplexes at film state.

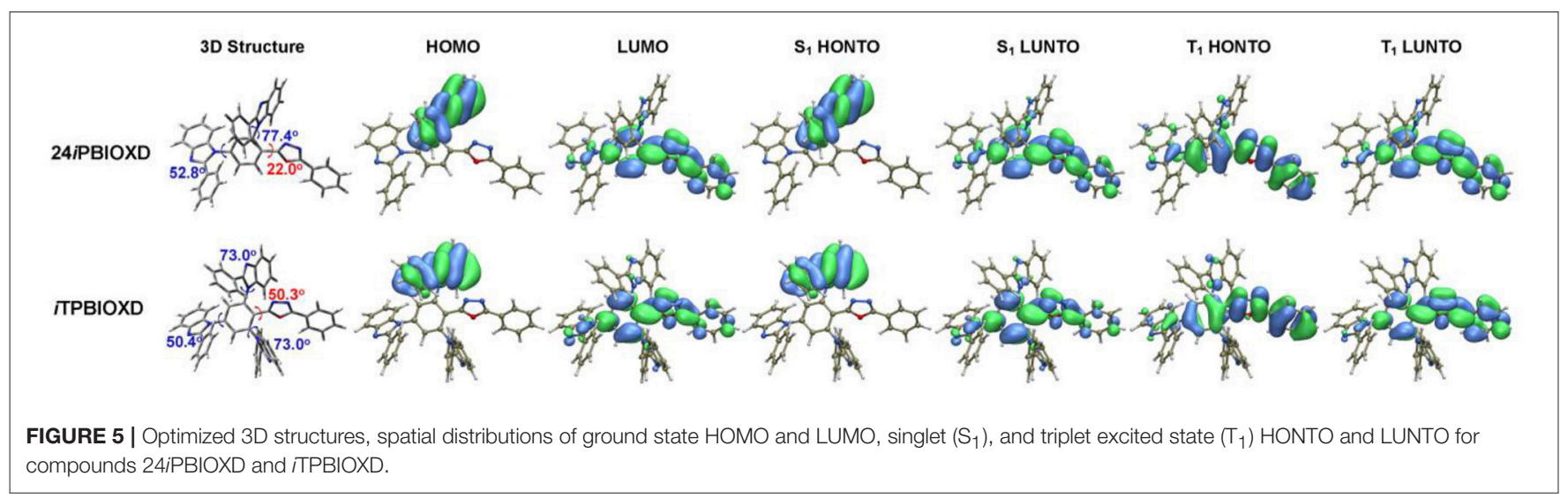

the penta-heterocyclic imidazoles, suggesting the weak electronwithdrawing property to gently participate electron-transport for the imidazoles. Similar distribution can be observed in the highest occupied natural transition orbital (HONTO) and the lowest unoccupied natural transition orbital (LUNTO) at singlet excited state. It should be noted that the HONTO distribution at triplet excited state was completely different from $S_{0}$ and $S_{1}$ for both compounds, which was mainly delocalized through the 2,5-diphenyl-1,3,4-oxadiazole skeleton, similar with the LUNTO distribution.

The electrochemical features were measured by cyclic voltammetry (CV) (Figure 6). Both compounds exhibited reversible reduction whereas undetectable oxidation behavior. The LUMO energy levels calculated from the onset of reduction curves for 24iPBIOXD and $i$ TPBIOXD were measured to be -2.80 and $-2.95 \mathrm{eV}$, while the HOMO energy levels calculated from the different between the LUMO and optical bandgaps $\left(E_{\mathrm{g}}\right)$ were evaluated to be -6.14 and $-6.17 \mathrm{eV}$, respectively. The values were in good agreement with the theoretical calculation. Besides, the energy levels for electron donor materials were also measured, with HOMO estimated from the onset of electrooxidation curves and LUMO calculated from HOMO and optical bandgaps. The HOMO/LUMO energy level values for mCP, TAPC, and TCTA were $-5.67 /-2.20,-5.05 /-1.61$, and $-5.19 /-1.92 \mathrm{eV}$, respectively. The deep HOMO and LUMO for the two new electron acceptors of 24iPBIOXD and $i$ TPBIOXD, provided sufficient driving forces on HOMO/LUMO energy offsets for the exciplex formation (Figure 6A). As shown in Figure 7A, the HOMO energy level offsets between the electron donor of TAPC, TCTA, or $\mathrm{mCP}$ and the electron acceptors of $24 i \mathrm{PBIOXD} / \mathrm{iTPBIOXD}$ were calculated to be $1.09 / 1.12$, $0.95 / 0.98$, or $0.47 / 0.5 \mathrm{eV}$, and the corresponding LUMO offsets were $1.19 / 1.34,0.88 / 1.03$, or $0.6 / 0.75 \mathrm{eV}$, respectively. It is noted in both acceptor systems, the TAPC donor based exciplex presented the highest driving force, followed by TCTA, while the $\mathrm{mCP}$ donor demonstrated the lowest HOMO/LUMO offsets.

\section{Electroluminescence Properties}

To investigate the charge transport properties of the two new $N$ linked isomeric benzoimidazole containing electron acceptors, single carrier electron-only device was prepared to find out the electron inject and transport properties of $24 i \mathrm{PBIOXD}$ and $i$ TPBIOXD. The device structure was ITO/24iPBIOXD, $i$ TPBIOXD, or TPBI $(50 \mathrm{~nm}) / \mathrm{LiF}(1 \mathrm{~nm}) / \mathrm{Al}(150 \mathrm{~nm})$, where the commercial electron transport material of $2,2,2-(1,3,5-$ phenylene)-tris(1-phenyl-1H-benzoimidazole) (TPBI) with $C$ linkage in benzoimidazole was selected for comparison. As shown in Figure 8, at the same operating voltage, TPBI based device exhibited the highest current density among all the three devices. Since the LUMO energy of TPBI $(2.7-2.9 \mathrm{eV})$ 

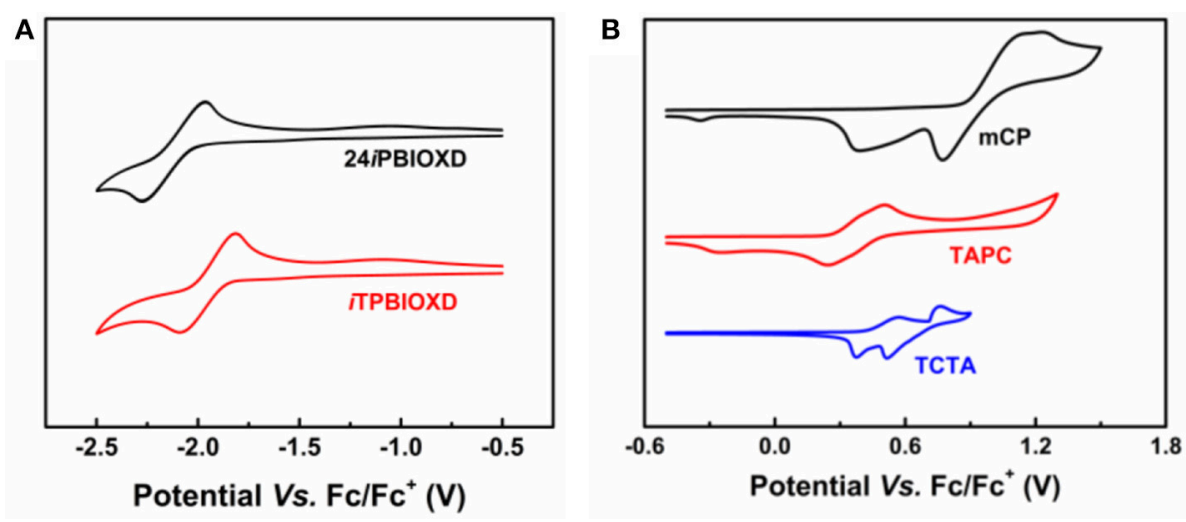

FIGURE 6 | Cyclic voltammograms of (A) 24iPBIOXD and ITPBIOXD in THF solution for reduction scan; (B) conventional electron donors (mCP, TAPC, and TCTA) in $\mathrm{CH}_{2} \mathrm{Cl}_{2}$ solution for oxidation scan.

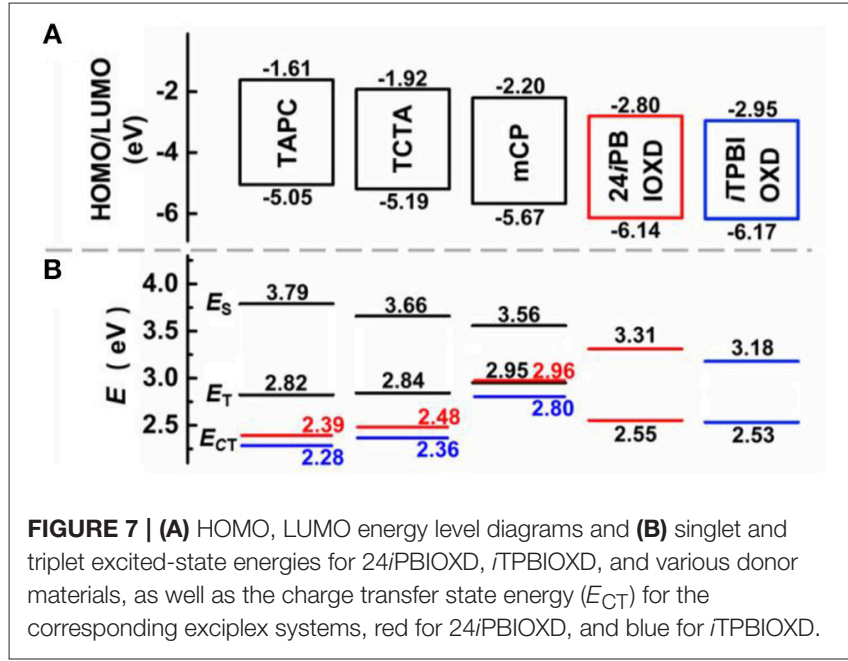

(Bian et al., 2018; Jou et al., 2018) was almost the same as 24iPBIOXD and $i$ TPBIOXD, which manifested their similar injection barrier for efficient electron injection. Therefore, the significantly higher current for TPBI indicated better electron transporting property than 24iPBIOXD and $i$ TPBIOXD. On the other hand, the current density in $i$ TPBIOXD device was slightly higher than 24iPBIOXD, as depicted in Figure 8, the LUMO level of $i$ TPBIOXD was $0.15 \mathrm{eV}$ lower than 24iPBIOXD, therefore a mildly efficient electron-injection could be attained in iTPBIOXD device due to its lower injection barriers. Thus, the electron-transport performance for both electron acceptors may be comparable.

To conduct a comprehensive comparison on the EL performance for the exciplex-TADF OLEDs among diverse electron-donor and acceptor systems, a series of vacuum deposited devices A-F were fabricated. Due to the highest HOMO level of TAPC for efficient hole-injection, the device configuration for TAPC-based OLEDs was ITO/ $\mathrm{MoO}_{3} \quad(1 \mathrm{~nm}) / \mathrm{TAPC}: 24 i \mathrm{PBIOXD}$ or $i$ TPBIOXD (1:1, $70 \mathrm{~nm}) / \mathrm{LiF}(1 \mathrm{~nm}) / \mathrm{Al}(100 \mathrm{~nm})$. To reduce the hole-injection

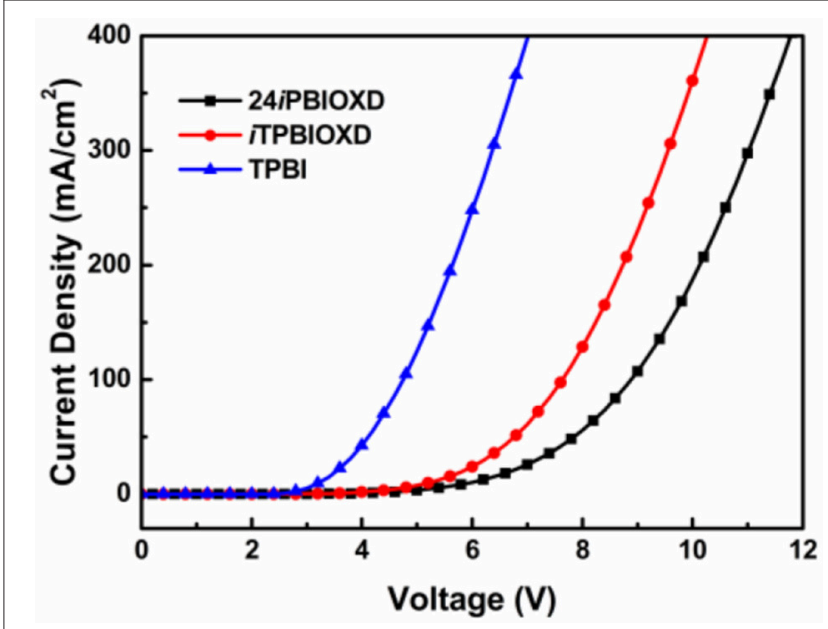

FIGURE 8 | J-V characteristic of nominal single-electron-only devices based on compounds 24iPBIOXD, ITPBIOXD, and TPBI [device structures: ITO/EML $(50 \mathrm{~nm}) / \mathrm{LiF}(1 \mathrm{~nm}) / \mathrm{Al}(150 \mathrm{~nm})]$.

barrier, TCTA-based device was constructed by ITO/ $\mathrm{MoO}_{3}$ $(1 \mathrm{~nm}) /$ TAPC $(40 \mathrm{~nm}) / \mathrm{TCTA}: 24 i \mathrm{PBIOXD}$ or $i$ TPBIOXD (1:1, $30 \mathrm{~nm}) / \mathrm{TmPyPB}(40 \mathrm{~nm}) / \mathrm{LiF} \quad(1 \mathrm{~nm}) / \mathrm{Al} \quad(100 \mathrm{~nm})$, while a further $10 \mathrm{~nm}$ TCTA thin film was inserted between the TAPC layer and emissive layer (EML) in mCP-based devices. Among them, $\mathrm{MoO}_{3}$ and $\mathrm{LiF}$ were used as hole- and electron-injection materials, respectively; TAPC and 1,3,5-tri[(3-pyridyl)-phen3-yl] benzene (TmPyPB) were functionalized as hole- and electron-transport materials, respectively, an extra TCTA layer was aimed to promote the hole-injection and block the electrons.

The current density-voltage-luminance $(J-V-L)$, electroluminescence (EL) spectra, together with the current and power efficiency, external quantum efficiency vs. luminance curves are shown in Figure 9. The device fabrication details are stated in Supporting Information. According to the key EL data listed in Table 2, the turn-on voltage for TAPC, TCTA, and $\mathrm{mCP}$ containing devices was gradiently increased from 2.8, 3.0 

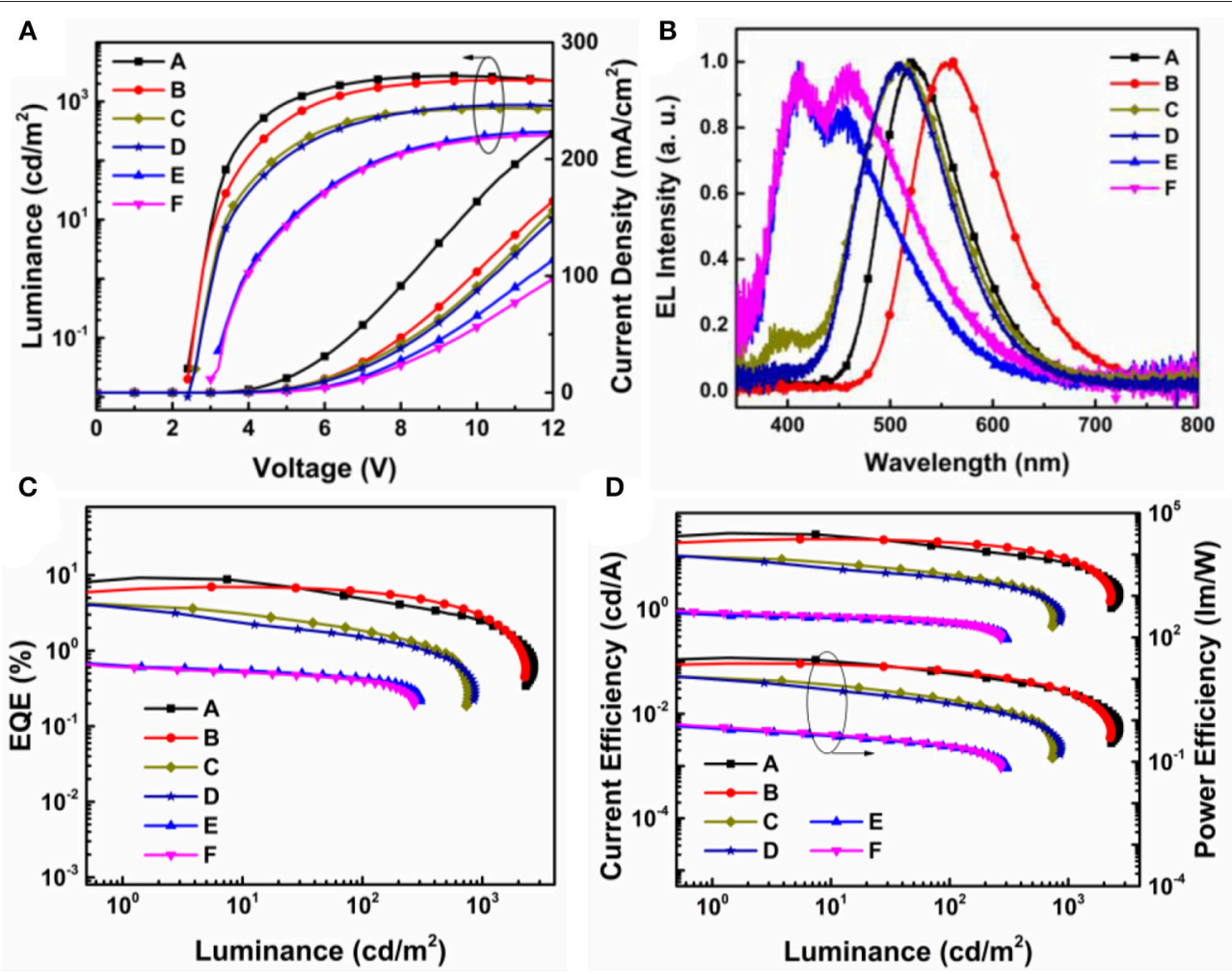

FIGURE 9 | (A) L-V-J characteristics; (B) normalized electroluminescent (EL) spectra; (C) external quantum efficiency (EQE) vs. luminance curves; (D) current efficiency and power efficiency vs. luminance curves of device A-F.

TABLE 2 | Electroluminescence characteristics for the devices.

\begin{tabular}{|c|c|c|c|c|c|c|}
\hline Device & Emitting layer & $v_{\text {on }}$ a $(\mathrm{V})$ & $\eta_{\mathrm{c}} \mathrm{b}(\mathrm{cd} / \mathrm{A})$ & $\begin{array}{c}\eta_{\mathrm{p}} \mathrm{c} \\
(\mathrm{Im} / \mathrm{W})\end{array}$ & $\eta_{\text {ext }} d(\%)$ & $\operatorname{CIE}(x, y)$ \\
\hline A & TAPC: 24iPBIOXD & 2.8 & 28.8 & 32.3 & 9.3 & $(0.31,0.55)$ \\
\hline C & TCTA: 24iPBIOXD & 3.0 & 10.1 & 10.6 & 4.0 & $(0.26,0.43)$ \\
\hline D & TCTA: ITPBIOXD & 3.0 & 10.6 & 10.2 & 3.9 & $(0.25,0.44)$ \\
\hline E & mCP: 24iPBIOXD & 3.8 & 0.8 & 0.66 & 0.65 & $(0.19,0.20)$ \\
\hline
\end{tabular}

${ }^{a}$ Turn-on voltage at $1 \mathrm{~cd} / \mathrm{m}^{2}$.

${ }^{b}$ Maximum current efficiency.

${ }^{c}$ Maximum power efficiency.

${ }^{d}$ Maximum external quantum efficiency.

to $3.8 \mathrm{eV}$. The as high as $0.48 \mathrm{eV}$ hole-injection barrier between TCTA and $\mathrm{mCP}$ lead to the highest operating voltage in devices $\mathrm{E}$ and $\mathrm{F}$. The EL performance trend was in consistent with the values of HOMO/LUMO energy offsets, and TAPC-analog bearing the highest driving forces for convenient exciplex formation demonstrated the best highest EL efficiency. The best performance was attained from device A with TAPC:24iPBIOXD exciplex, corresponding to a maximum current efficiency (CE), power efficiency $(\mathrm{PE})$, and external quantum efficiency (EQE) of $28.8 \mathrm{~cd} / \mathrm{A}, 32.3 \mathrm{~lm} / \mathrm{W}$, and $9.3 \%$. And the TAPC:iTPBIOXD based device $\mathrm{B}$ demonstrated slightly poorer EL performance with maximum current efficiency, power efficiency and external quantum efficiency of $22.1 \mathrm{~cd} / \mathrm{A}, 23.41 \mathrm{~m} / \mathrm{W}$, and $7.0 \%$, respectively. Device C and D based on TCTA electron donor showed comparable EL efficiency, with maximum EQE of 4.0 and $3.9 \%$ for $24 i \mathrm{PBIOXD}$ and $i$ TPBIOXD electron acceptors, respectively. The EL performance for $\mathrm{MCP}$ device was rather poor, with maximum EQE of $<1 \%$ in both device $\mathrm{E}$ and $\mathrm{F}$. As depicted in Figure 9B, devices A and B with TAPC donor depicted smooth exciplex-TADF emission, with EL peak at 519 and $556 \mathrm{~nm}$, respectively, which is in agreement with the relevant PL spectra. Commission Internationale de L'Eclairage (CIE) values for device $\mathrm{A}$ and $\mathrm{B}$ was measured at $(0.31,0.55)$ and $(0.43,0.54)$, corresponding to green and yellow emission, 
respectively. The TCTA based device C and D both exhibited blueish-green emission, with a gentle shoulder peak at around $400 \mathrm{~nm}$ for $24 i \mathrm{PBIOXD}$. The two mCP-based devices displayed blue emission with CIE $\mathrm{x}, \mathrm{y}$ each at $\sim 0.20$. However, the EL spectra of device $\mathrm{E}$ and $\mathrm{F}$ revealed bimodal emission showing comparable intensity for the two peaks. It is hypothesized that the inadequate HOMO and LUMO energy offsets $(<\sim 1 \mathrm{eV})$ for TCTA:24iPBIOXD, mCP:24iPBIOXD, and mCP:iTPBIOXD, resulted in the unexpected shorter wavelength EL emission peak, which was ascribed to pure mCP emission (Chiu and Lee, 2012; Shahalizad et al., 2017). In addition, since the $E_{\mathrm{CT}}$ of TAPC and TCTA based exciplexes were lower than the triplet energy of both donor and acceptor materials, which was beneficial to restrict triplet excitons in the exciplex states for the efficient RISC. However, $E_{\mathrm{CT}}$ of 2.8-2.96 eV (Figure 7B) for $\mathrm{mCP}$ based exciplex was significantly higher than the triplet energy $(\sim 2.55 \mathrm{eV})$ of the two electron acceptors, which provided a potential way for energy leakage from exciplex states to the $\mathrm{T}_{1}$ excited state of $24 i \mathrm{PBIOXD}$ and $i$ TPBIOXD. Thus, devices based on $\mathrm{mCP}$ donors demonstrated the lowest EL efficiency and inadequate TADF emission.

\section{CONCLUSION}

In summary, we have designed and synthesized two universal $N$-linked benzoimidazole/oxadiazole hybrid electron acceptors through a simple nucleophilic substitution reaction. Diverse deep-blue to yellow emissive exciplex could be formed between various conventional donor materials and the two acceptors due to their deep HOMO levels of $\sim 6.15 \mathrm{eV}$. The HOMO and LUMO energy level offsets which were also named as the driving forces for exciplex formation were gradiently increased from 0.47 to 1.12 and 0.6 to $1.34 \mathrm{eV}$ in $\mathrm{mCP}$, TCTA, to TAPC based exciplexes. We have found that both HOMO and LUMO offsets $\geq 1 \mathrm{eV}$ was required to form efficient and stable intermolecular charge transfer exciplex. When the driving forces were as low as

\section{REFERENCES}

Adachi, C., Baldo, M. A., Thompson, M. E., and Forrest, S. R. (2001). Nearly 100\% internal phosphorescence efficiency in an organic light-emitting device. J. Appl. Phys. 90, 5048-5051. doi: 10.1063/1.1409582

Baldo, M. A., O’Brien, D. F., Thompson, M. E., and Forrest, S. R. (1999). Excitonic singlet-triplet ratio in a semiconducting organic thin film. Phys. Rev. B 60, 14422-14428. doi: 10.1103/PhysRevB.60.14422

Baldo, M. A., O’Brien, D. F., You, Y., Shoustikov, A., Sibley, S., Thompson, M. E., et al. (1998). Highly efficient phosphorescent emission from organic electroluminescent devices. Nature 395, 151-154. doi: 10.1038/ 25954

Becke, A. D. (1988). Density-functional exchange-energy approximation with correct asymptotic behavior. Phys. Rev. A 38, 3098-3100. doi: 10.1103/PhysRevA.38.3098

Bian, M., Wang, Y., Guo, X., Lv, F., Chen, Z., Duan, L., et al. (2018). Positional isomerism effect of spirobifluorene and terpyridine moieties of "(A)n-D-(A)n" type electron transport materials for long-lived and highly efficient TADF-PhOLEDs. J. Mater. Chem. C 6, 10276-10283. doi: 10.1039/c8tc $03796 \mathrm{e}$
0.47-0.75 eV, which is far $<1 \mathrm{eV}$, the two mCP based exciplex demonstrated considerably short delayed component lifetime, with values of only 42 ad 72 ns for $24 i \mathrm{PBIOXD}$ and $i$ TPBIOXD acceptors, respectively. Additionally, the exciplex-type device EQE was lower than $1 \%$. When the driving forces were slightly lower or approaching $1 \mathrm{eV}$, the two TCTA exciplexes displayed moderate EL efficiency of about $4 \%$. And the best EL performance was achieved in TAPC containing exciplex-type TADF OLEDs, with relatively low turn-on voltage of $2.8 \mathrm{~V}$, maximum efficiency of $28.8 \mathrm{~cd} / \mathrm{A} \mathrm{CE}, 32.3 \mathrm{~lm} / \mathrm{W} \mathrm{PE}$, and $9.3 \%$ for 24 PBIOXD acceptor and $22.1 \mathrm{~cd} / \mathrm{A} \mathrm{CE}, 23.4 \mathrm{~lm} / \mathrm{W}$ and $7.0 \%$ for $i \mathrm{TPBIOXD}$ acceptor. Our results provide guidance on the exploration of efficient exciplex type TADF OLEDs.

\section{AUTHOR CONTRIBUTIONS}

WY, MZ, and DH designed and synthesized the materials. WY and MZ did most of the experimental work and data analyses. OLED device fabrication and electroluminescent performance studies were carried out by HY and NS. YT had the idea, led the project. WY and YT prepared the manuscript. All authors contributed to the manuscript preparation.

\section{ACKNOWLEDGMENTS}

We declare all sources of funding received for the research being submitted. We thank the National Natural Science Foundation of China (91833304 and 61805211), the Natural Science Foundation of Jiangsu Province (BK20160042 and XYDXX-026) for financial support.

\section{SUPPLEMENTARY MATERIAL}

The Supplementary Material for this article can be found online at: https://www.frontiersin.org/articles/10.3389/fchem. 2019.00187/full\#supplementary-material 
Francl, M. M., Pietro, W. J., Hehre, W. J., Binkley, J. S., Gordon, M. S., DeFrees, D. J., et al. (1982). Self-consistent molecular orbital methods. XXIII. A polarization-type basis set for second-row elements. J. Chem. Phys. 77, 3654-3665. doi: 10.1063/1.444267

Gong, S., Chen, Y., Yang, C., Zhong, C., Qin, J., and Ma, D. (2010). De Novo design of silicon-bridged molecule towards a bipolar host: all-phosphor white organic light-emitting devices exhibiting high efficiency and low efficiency roll-off. $A d v$. Mater. 22, 5370-5373. doi: 10.1002/adma.201002732

Goushi, K., and Adachi, C. (2012). Efficient organic light-emitting diodes through up-conversion from triplet to singlet excited states of exciplexes. Appl. Phys. Lett. 101:023306. doi: 10.1063/1.4737006

Goushi, K., Yoshida, K., Sato, K., and Adachi, C. (2012). Organic light-emitting diodes employing efficient reverse intersystem crossing for triplet-to-singlet state conversion. Nat. Photon. 6, 253-258. doi: 10.1038/nphoton.2012.31

Hu, J., Zhao, C., Zhang, T., Zhang, X., Cao, X., Wu, Q., et al. (2017). Isomeric Nlinked benzoimidazole containing new electron acceptors for exciplex forming hosts in highly efficient blue phosphorescent OLEDs. Adv. Optical Mater. 5:1700036. doi: 10.1002/adom.201700036

Huang, T., Jiang, W., and Duan, L. (2018). Recent progress in solution processable TADF materials for organic light-emitting diodes. J. Mater. Chem. C 6, 5577-5596. doi: 10.1039/C8TC01139G

Hung, W.-Y., Chiang, P.-Y., Lin, S.-W., Tang, W.-C., Chen, Y.-T., Liu, S.H., et al. (2016). Balance the carrier mobility to achieve high performance exciplex OLED using a triazine-based acceptor. ACS Appl. Mater. Interfaces 8, 4811-4818. doi: 10.1021/acsami.5b11895

Hung, W.-Y., Wang, T.-C., Chiang, P.-Y., Peng, B.-J., and Wong, K.T. (2017). Remote steric effect as a facile strategy for improving the efficiency of exciplex-based OLEDs. ACS Appl. Mater. Interfaces 9, 7355-7361. doi: 10.1021/acsami.6b16083

Jankus, V., Data, P., Graves, D., McGuinness, C., Santos, J., Bryce, M. R., et al. (2014). Highly efficient TADF OLEDs: how the emitter-host interaction controls both the excited state species and electrical properties of the devices to achieve near 100\% triplet harvesting and high efficiency. Adv. Funct. Mater. 24, 6178-6186. doi: 10.1002/adfm.201400948

Jeon, S. K., Yook, K. S., and Lee, J. Y. (2016). Highly efficient exciplex organic light-emitting diodes using thermally activated delayed fluorescent emitters as donor and acceptor materials. Nanotechnology 27:224001. doi: 10.1088/0957-4484/27/22/224001

Jou, J.-H., Weng, J.-W., Chavhan, S. D., Yadav, R. A. K., and Liang, T.-W. (2018). Investigation of charge-transporting layers for highefficiency organic light-emitting diode. J. Phys. D: Appl. Phys. 51:454002. doi: 10.1088/1361-6463/aad951

Lee, C., Yang, W., and Parr, R. G. (1988). Development of the Colic-Salvetti correlation-energy formula into a functional of the electron density. Phys. Rev. B 37, 785-789. doi: 10.1103/PhysRevB.37.785

Lee, J.-H., Cheng, S.-H., Yoo, S.-J., Shin, H., Chang, J.-H., Wu, C.-I., et al. (2015). An exciplex forming host for highly efficient blue organic light emitting diodes with low driving voltage. Adv. Funct. Mater. 25, 361-366. doi: 10.1002/adfm.201402707

Li, J., Ding, D., Tao, Y., Wei, Y., Chen, R., Xie, L., et al. (2016). A significantly twisted spirocyclic phosphine oxide as a universal host for high-efficiency full-color thermally activated delayed fluorescence diodes. Adv. Mater. 28, 3122-3130. doi: 10.1002/adma.201506286

Lin, T.-A., Chatterjee, T., Tsai, W.-L., Lee, W.-K., Wu, M.-J., Jiao, M., et al. (2016). Sky-blue organic light emitting diode with $37 \%$ external quantum efficiency using thermally activated delayed fluorescence from spiroacridine-triazine hybrid. Adv. Mater. 28, 6976-6983. doi: 10.1002/adma.201601675

Liu, W., Chen, J.-X., Zheng, C.-J., Wang, K., Chen, D.-Y., Li, F., et al. (2016). Novel strategy to develop exciplex emitters for high-performance OLEDs by employing thermally activated delayed fluorescence materials. Adv. Funct. Mater. 26, 2002-2008. doi: 10.1002/adfm.201505014

Liu, X.-K., Chen, Z., Qing, J., Zhang, W.-J., Wu, B., Tam, H. L., et al. (2015a). Remanagement of singlet and triplet excitons in single-emissive-layer hybrid white organic light-emitting devices using thermally activated delayed fluorescent blue exciplex. Adv. Mater. 27, 7079-7085. doi: 10.1002/adma.201502897

Liu, X.-K., Chen, Z., Zheng, C.-J., Liu, C.-L., Lee, C.-S., Li, F., et al. (2015b). Prediction and design of efficient exciplex emitters for high-efficiency, thermally activated delayed-fluorescence organic light-emitting diodes. $A d v$. Mater. 27, 2378-2383. doi: 10.1002/adma.201405062

Liu, Y., Li, C., Ren, Z., Yan, S., and Bryce, M. R. (2018). All-organic thermally activated delayed fluorescence materials for organic light-emitting diodes. Nat. Rev. Mater. 3:18020. doi: 10.1038/natrevmats.2018.20

Liu, Z., Qayyum, M. F., Wu, C., Whited, M. T., Djurovich, P. I., Hodgson, K. O., et al. (2011). A codeposition route to cui-pyridine coordination complexes for organic light-emitting diodes. J. Am. Chem. Soc. 133, 3700-3703. doi: $10.1021 /$ ja1065653

Lo, S.-C., Harding, R. E., Shipley, C. P., Stevenson, S. G., Burn, P. L., and Samuel, I. D. (2009). High-triplet-energy dendrons: enhancing the luminescence of deep blue phosphorescent iridium(III) complexes. J. Am. Chem. Soc. 131, 16681-16688. doi: 10.1021/ja903157e

Ma, Y., Zhang, H., Shen, J., and Che, C. (1998). Electroluminescence from triplet metal-ligand charge-transfer excited state of transition metal complexes. Synth. Met. 94, 245-248. doi: 10.1016/S0379-6779(97)04166-0

Mondal, E., Hung, W.-Y., Dai, H.-C., and Wong, K.-T. (2013). Fluorenebased asymmetric bipolar universal hosts for white organic light emitting devices. Adv. Funct. Mater. 23, 3096-3105. doi: 10.1002/adfm.2012 02889

Oh, C. S., Kang, Y. J., Jeon, S. K., and Lee, J. Y. (2015). High efficiency exciplex emitters using donor-acceptor type acceptor material. J. Phys. Chem. C 119, 22618-22624. doi: 10.1021/acs.jpcc.5b05292

Olivier, Y., Moral, M., Muccioli, L., and Sancho-Garcíae, J.-C. (2017). Dynamic nature of excited states of donor-acceptor TADF materials for OLEDs: how theory can reveal structure-property relationships. J. Mater. Chem. C 5, 5718-5729. doi: 10.1039/C6TC05075A

Park, Y.-S., Lee, S., Kim, K.-H., Kim, S.-Y., Lee, J.-H., and Kim, J.-J. (2013). Exciplex-forming co-host for organic light-emitting diodes with ultimate efficiency. Adv. Funct. Mater. 23, 4914-4920. doi: 10.1002/adfm.2013 00547

Sarma, M., and Wong, K.-T. (2018). Exciplex: an intermolecular chargetransfer approach for TADF. ACS Appl. Mater. Interfaces 10, 19279-19304. doi: 10.1021/acsami.7b18318

Segal, M., Baldo, M. A., Holmes, R. J., Forrest, S. R., and Soos, Z. G. (2003). Excitonic singlet-triplet ratios in molecular and polymeric organic materials. Phys. Rev. B 68:075211. doi: 10.1103/PhysRevB.68.075211

Shahalizad, A., D’Aléo, A., Andraud, C., Sazzad, M. H., Kim, D.-H., Tsuchiya, Y., et al. (2017). Near infrared electroluminescence from $\mathrm{Nd}(\mathrm{TTA})_{3}$ phen in solution-processed small molecule organic light-emitting diodes. Org. Electron. 44, 50-58. doi: 10.1016/j.orgel.2017.01.044

Son, K. S., Yahiro, M., Imai, T., Yoshizaki, H., and Adachi, C. (2008). Analyzing bipolar carrier transport characteristics of diarylamino-substituted heterocyclic compounds in organic light-emitting diodes by probing electroluminescence spectra. Chem. Mater. 20, 4439-4446. doi: 10.1021/cm8004985

Su, H.-C., Chen, H.-F., Fang, F.-C., Liu, C.-C., Wu, C.-C., Wong, K.-T., et al. (2008). Solid-state white light-emitting electrochemical cells using iridiumbased cationic transition metal complexes. J. Am. Chem. Soc. 130, 3413-3419. doi: $10.1021 / \mathrm{ja} 076051 \mathrm{e}$

Sun, J. W., Lee, J.-H., Moon, C.-K., Kim, K.-H., Shin, H., and Kim, J.-J. (2014). A fluorescent organic light-emitting diode with $30 \%$ external quantum efficiency. Adv. Mater. 26, 5684-5688. doi: 10.1002/adma.201401407

Tang, C. W., and VanSlyke, S. A. (1987). Organic electroluminescent diodes. Appl. Phys. Lett. 51, 913-915. doi: 10.1063/1.98799

Tao, Y., Yang, C., and Qin, J. (2011). Organic host materials for phosphorescent organic light-emitting diodes. Chem. Soc. Rev. 40, 2943-2970. doi: $10.1039 / \mathrm{c} 0 \mathrm{cs} 00160 \mathrm{k}$

Uoyama, H., Goushi, K., Shizu, K., Nomura, H., and Adachi, C. (2012). Highly efficient organic light-emitting diodes from delayed fluorescence. Nature 492 234-238. doi: 10.1038/nature11687

Volz, D., Zink, D. M., Bocksrocker, T., Friedrichs, J., Nieger, M., Baumann, T., et al. (2013). Molecular construction kit for tuning solubility, stability and luminescence properties: heteroleptic MePyrPHOS-copper iodide-complexes and their application in organic light-emitting diodes. Chem. Mater. 25, 3414-3426. doi: 10.1021/cm4010807

Wen, S. W., Lee, M. T., and Chen, C. H. (2005). Recent development of blue fluorescent OLED materials and devices. J. Disp. Technol. 1, 90-99. doi: 10.1109/JDT.2005.852802 
Wu, Q., Wang, M., Cao, X., Zhang, D., Sun, N., Wan, S., et al. (2018). Carbazole/ $\alpha-$ carboline hybrid bipolar compounds as electron acceptors in exciplex or nonexciplex mixed cohosts and exciplex-TADF emitters for high-efficiency OLEDs. J. Mater. Chem. C 6, 8784-8792. doi: 10.1039/C8TC02353K

Wu, T.-L., Huang, M.-J., Lin, C.-C., Huang, P.-Y., Chou, T.-Y., and Cheng, R.W., et al. (2018). Diboron compound-based organic light-emitting diodes with high efficiency and reduced efficiency roll-off. Nat. Photon. 12, 235-240. doi: 10.1038/s41566-018-0112-9

Yao, C., Yang, Y., Li, L., Bo, M., Peng, C., and Wang, J. (2018). Ge-based bipolar small molecular host for highly efficient blue OLEDs: multiscale simulation of charge transport. J. Mater. Chem. C 6, 6146-6152. doi: 10.1039/C8TC0 $0355 \mathrm{~F}$

Zeng, W., Lai, H.-Y., Lee, W.-K., Jiao, M., Shiu, Y.-J., Zhong, C., et al. (2018). Achieving nearly $30 \%$ external quantum efficiency for orange-red organic light emitting diodes by employing thermally activated delayed fluorescence emitters composed of 1,8-naphthalimide-acridine hybrids. Adv. Mater. 30:1704961. doi: 10.1002/adma.201704961

Zhang, D., Cai, M., Bin, Z., Zhang, Y., Zhang, D., and Duan, L. (2016). Highly efficient blue thermally activated delayed fluorescent OLEDs with record-low driving voltages utilizing high triplet energy hosts with small singlet-triplet splittings. Chem. Sci. 7, 3355-3363. doi: 10.1039/C5SC04755B

Zhang, D., Cao, X., Wu, Q., Zhang, M., Sun, N., Zhang, X., et al. (2018). Purely organic materials for extremely simple all-TADF white OLEDs: a new carbazole/oxadiazole hybrid material as a dual-role non-doped light blue emitter and highly efficient orange host. J. Mater. Chem. C 6, 3675-3682. doi: 10.1039/C7TC04969B

Zhang, L., Cai, C., Li, K. F., Tam, H. L., Chan, K. L., and Cheah, K. W. (2015). Efficient organic light-emitting diode through triplet exciton reharvesting by employing blended electron donor and acceptor as the emissive layer. ACS Appl. Mater. Interfaces 7, 24983-24986. doi: 10.1021/acsami.5b05597

Zhang, T., Zhao, B., Chu, B., Li, W., Su, Z., Wang, L., et al. (2015). Blue exciplex emission and its role as a host of phosphorescent emitter. Org. Electron. 24, 1-6. doi: 10.1016/j.orgel.2015.05.013

Zhang, Y., and Forrest, S. R. (2012). Triplets contribute to both an increase and loss in fluorescent yield in organic light emitting diodes. Phys. Rev. Lett. 108:267404. doi: 10.1103/PhysRevLett.108.267404

Conflict of Interest Statement: The authors declare that the research was conducted in the absence of any commercial or financial relationships that could be construed as a potential conflict of interest.

Copyright (c) 2019 Yuan, Yang, Zhang, Hu, Sun and Tao. This is an open-access article distributed under the terms of the Creative Commons Attribution License (CC $B Y)$. The use, distribution or reproduction in other forums is permitted, provided the original author(s) and the copyright owner(s) are credited and that the original publication in this journal is cited, in accordance with accepted academic practice. No use, distribution or reproduction is permitted which does not comply with these terms. 